WIENER SLAVISTISCHES JAHRBUCH, Band 55/2009, 139-144

(C) 2009 by Österreichische Akademie der Wissenschaften, Wien

L U D G E R U D O L P H

\title{
Bulgarische Literatur und Internet
}

\section{I}

Die Entwicklung des Internet bedeutet die Möglichkeit, an einem beliebigen Ort zu beliebiger Zeit Informationen in Wort, Bild und Ton abzurufen oder auszusenden, sofern man über einen PC mit Internetanschluss und - bei der Sendung von Informationen - auch über das nötige technische Wissen verfügt. Das ist auch in Europa inzwischen in großem Umfang der Fall. Die Revolutionierung der Informationstechnologie traf hier mit dem Ende der sowjetrussischen Herrschaft zusammen; was zugleich bedeutet, dass die politisch kontrollierte und gesteuerte Information an ihr Ende gelangt war. Das Weltnetz (world wide web) erscheint enthierarchisiert und dezentralisiert. Stärker noch als zuvor ist gerade im Netz die Menge der Informationen nicht mehr überschaubar. Wenn bei einer Bibliothek der Standort des Gebäudes, die Aufstellung der Bücher, der Katalog, der Bibliothekar u. ä. die Benutzung strukturieren und den Benutzer lenken, so ist es im Internet der Nutzer selber, der nach seinen - oft sogar nur augenblicklichen - Interessen das Angebot selber filtert und strukturiert (und u. U. sogar mitgestaltet). Im Surfen treffen dabei Neugier, Voyeurismus, der Wunsch nach Information und Unterhaltung zu oftmals zufälligen Funden und Entdeckungen zusammen.

\section{II}

Die bulgarische Literatur hat seit ihrer Entwicklung im frühen Mittelalter an den Etappen der Verschriftlichung in Handschriften (seit dem 10. Jahrhundert) und sehr spät: 1806 - im Buchdruck, dann seit der 2. Hälfte des 19. Jahrhunderts an der Ausdifferenzierung der literarischen Gattungen und an der Entwicklung von Massenmedien und des Buchmarkts teilgenommen. Und so ist es auch nur folgerichtig, wenn heute bulgarische Literatur auch im Internet zu finden ist. Das Literaturinstitut der Bulgarischen Akademie der Wissenschaften ${ }^{1}$ machte allerdings bisher keine

${ }^{1}$ Aufzurufen unter www.ilit.bas.bg (letzter Zugriff am 18. 10. 2006). 
Texte über das Internet zugänglich; offenbar liegt die Digitalisierung und Bereitstellung des ,kulturellen Erbes ‘ (Bücher, Handschriften, Nachlässe u. ä.) mit Hilfe eines modernen Mediums außerhalb ihres Interesses oder ihrer augenblicklichen Möglichkeiten. Gerade die digitale Edition mittelalterlicher Codices und sonstiger Handschriften wäre aber doch wohl eine wichtige Aufgabe der Akademie. Die Fachzeitschrift des Literaturinstituts, „Literaturna Misъl“, ist seit 1999 mit ihrem Inhaltsverzeichnis und ausgewählten Artikeln online. ${ }^{2}$ Zur Gänze frei zugänglich sind dagegen „Literaturni Balkani“, „Plamъk“, „Rodna reč“, „Literaturen vestnik“, „Teatъr“ und „Въlgarski ezik i literatura“ 3 Im Netz präsent sind bereits die bulgarischen Bibliotheken; offenbar planmäßig wird ein umfassender Verbund aufgebaut, dessen Bestände dann über den OPAC recherchierbar sind oder sein sollen; bei der Bibliothek der BAN ist dies bereits möglich. ${ }^{4}$

Sucht man nun bulgarische Literatur, ihre alten und neuen Texte, dann sieht man sich auf private Initiativen verwiesen. LiterNet, ${ }^{5}$ ein seit 1999 arbeitender elektronischer Verlag (den es auch für andere Literaturen gibt), ruft Autoren und Übersetzer auf, ihr e-Buch zu veröffentlichen, und kann dabei auf folgende Vorteile verweisen: keine bürokratischen Hindernisse, keine hohen Preise und keine Schwierigkeiten des traditionellen Buchvertriebs; kürzester Weg zum Leser - in Bulgarien und weltweit; Veröffentlichung innerhalb eines Monats. ${ }^{6}$ Als Redakteur zeichnet Georgi Čobanov verantwortlich, der seine „Kurze Einführung in die bulgarische Literatur“ mit dem programmatischen Satz beschließt: Diese Literatur eines kleinen Volkes bewähre sich auch jetzt, ,da uns das Internet als globale Media [sic] die Chance zu einem Anschluss an die Weltliteratur ermöglicht". ${ }^{7}$ LiterNet veröffentlicht literarische und wissenschaftliche Texte zu Literatur und Philosophie; das Spektrum der literarischen Gattungen ist recht breit: Lyrik, Kurzprosa, Erzählungen, dramatische Werke, Filmszenarien, Essays. Es finden sich sogar 21 bulgarische Romane (im Original und/ oder in Übersetzung), - eine im Internet sicher nur schwierig zu vermittelnde Gattung, denn die Lektüre umfangreicher Werke - und seien sie auch noch so spannend - dürfte auf einem PC, den man ja nicht bequem zur Hand nehmen kann, kaum zu leisten sein. Die elektronisch edierten Bücher sind allerdings auch online über die ,knižarnica“ von LiterNet zu erwerben. Der Schwerpunkt von LiterNet liegt auf den

2 http://ilit.bas.bg/litmis (letzter Zugriff am 18. 10. 2006).

3 Zugänglich über die Website von Slovoto: www.slovo.bg; „Bъlgarski ezik i literatura“ über LiterNet $>$ E-spisanie BEL.

4 Aufzurufen unter cl.bas.bg; die Website „Bibliotekite v Bъlgarija“ ist aufzurufen unter bibliotekite.bg (19. 10. 2006).

5 http://www.liternet.bg; zu sechs bulgarischen Websites, deren Inhalt die bulgarische Literatur ist (ilit.bas.bg; hulite.net; slovo.bg; litclub.com; welkya.com und liternet.bg) gelangt man auch über die Seite der Dichterin Roumena Schindler-Kolarowa: de.roumena. net.

${ }^{6}$ LiterNet > Izdatelstvo > Kak da publikuvame v „LiterNet“".

7 Georgi Chobanov, Kurze Einführung in die bulgarische Literatur, aus dem Bulg. von Ludmilla Eimer: www.ludmilla-eimer.de (25. 10. 2006). 
Autoren und Autorinnen der Gegenwart (aufzurufen unter ,avtori'), die dieses Medium offenbar auch gern nutzen; gelegentlich trifft man auch auf einen ,Klassiker“ (Vazov, A. Konstantinov, Javorov, Trajanov u. a.). Zu den digitalisierten Autoren, darunter auch zahlreiche nichtbulgarische, sind in der Regel auch essayistisch-kritische Arbeiten abrufbar. Sucht man ältere und klassische bulgarische Literatur, so sei am ehesten auf „Slovoto. Bъlgarska virtualna biblioteka“ verwiesen, ${ }^{8}$ eine Unternehmung von 39 idealistischen Männern und Frauen verschiedenen Alters und verschiedener Profession, die durch ihre Liebe zur Literatur und zum Lesen verbunden sind. ${ }^{9}$ „Виртуална библиотека ,Словото“ си е поставила за цел да публикува в интернет всички значими произведения на българската литература““. ${ }^{10}$ Hier findet man über den Link ,avtori“ Vazovs „Pod igoto“ ebenso wie seine „Chъšove“, Dimovs „Tjutjun“, Javorovs Dramen und Gedichte, Lilievs „Ptici v noštta“, Vapcarov, einiges von Trajanov, Botev, Karavelov, Paisij, Petko und Penčo Slavejkov, Prličev in bulgarischer Übersetzung, Michajlovski, Veličkov. Aus der alten Literatur findet man bisher allerdings nur die „Azbučna molitva“ von Konstantin Preslavski (in bulgarischer, altkirchenslavischer und englischer Sprache). Die Nachteile solcher ,Leseausgaben' sind schnell spürbar: Es fehlen ein textkritischer Kommentar, Anmerkungen, Zeugnisse; in der wissenschaftlichen Forschung ist man also nach wie vor auf die Buchausgaben - sofern vorhanden - angewiesen.

Für Debütanten hat Slovoto eine eigene website eingerichtet: Chulite. ${ }^{11}$ „Това е сайт за нова българска Художествена Литература. Създаден е с цел да помогне на нови и неутвърдени автори да публикуват своите произведения, да даде възможност за комуникация между тях и същевременно да запознае любителите на художественото слово с техните творби“. ${ }^{12}$ Die ,razdeli“ in der ,Chuliteka“ sind: ,Razkazi“, ,Romani“, ,Izbrano ljubovna lirika', ,Izbrano proza', ,Izbrano poezija‘, ,Ljubovna lirika' (die mit 1.280.985 Aufrufen offenbar beliebteste Abteilung), ,Prikazki`, ,Proizvedenija za deca‘, ,Proizvedenija na čužd ezik‘ (nur englische Texte, irrtümlich hat sich ein bulgarischer eingeschlichen), ,Prevodi', ,Poezija“, ,Nъ čis bъlgarski‘ (sic), ,Chajku‘, ,Fantastika', ,Chumor i satira‘, ,sms-poezija‘, ,Erotika‘, ,Eseta“, ,Pъtepisi‘ und ,Drugi ...‘. Eine Analyse dieser Texte - wenn dies bei der (Un)menge der Texte überhaupt noch möglich sein sollte - steht noch aus.

III

Mit der Etablierung des Internet begann auch in Bulgarien die theoretische Auseinandersetzung mit diesem neuen Medium. Georgi Čobanov, der Schöpfer von LiterNet, hat wohl aus einer Art Aufbruchstimmung heraus emphatisch seine Meinung

\footnotetext{
8 www.slovo.bg.

9 Vgl. den link,za nas“ (letzter Zugriff 18. 10. 2006).

${ }^{10}$ So der Text auf der Hauptseite: www.slovo.bg (19. 10. 2006).

11 Aufzurufen über den Link, vrъzki ‘ bei Slovoto oder direkt über www.hulite.net.

12 So auf der Eingangsseite: www.hulite.net (19. 10. 2006).
} 
über Literatur im Internet artikuliert, die er als Auflösung traditioneller, gleichsam ,vordigitaler Herrschaftsbeziehungen ' sieht. ${ }^{13}$ Literarische Institutionen, d. h. Schulen, Universitäten, der literarische Kanon, bilden den ,ogromen simvoličen i disciplinirašt potencial“", der die literarischen Hierarchien festlegt und für die Rezipienten vorschreibt. Dieses Autoritätsverhältnis werde das Internet (,edno decentrirano literaturno prostranstvo“) beenden: „В крайна сметка свободата в Мрежата е точно това - не да преповтори преддигиталната йерархия от ценности, не и да създаде паралелна литературна йерархия чрез ,мрежовите“ автори и жанрове, а да остави процеса отворен за взаимопроникване“. Im Internet können die „Netzautoren“ und die „Netzleser“ ohne Zwischeninstitutionen wie Wissenschaftler und Kritiker in direkten Austausch treten, leicht und schnell kann der Leser resp. Surfer selber zum Autor werden. Das Internet bewirke daher, so Čobanov, 1) eine ,радикална промяна на начините за представяне на текста“ und 2) eine „радикална промяна в естеството и в създаването на самия художествен продукт и неговото възприемане“.

Mag in Čobanovs Theoretisieren noch der avantgardistisch anmutende Impetus der ,tabula rasa' zu verspüren sein, so sind die Ausführungen von Martin Mitov, dem Webdesigner von Slovoto, deutlich praxis- und erfahrungsorientiert. ${ }^{14}$ Das digitale Buch hat die traditionelle Papierform nicht verdrängen können, dasselbe gilt auch für Zeitschriften und Zeitungen. Obwohl der mit einem Preis ausgezeichnete und von der Kritik hochgelobte „Estestven roman“ von Georgi Gospodinov bereits fünf Jahre im Netz steht und hier praktisch kostenlos zu bekommen ist, sind beide Buchauflagen ausverkauft. ${ }^{15}$ Denn ein Buch ist praktikabel, gut zu handhaben, in ihm lesen kann man fast überall, ein Philologe kann mit mehreren Büchern gleichzeitig auf seinem Tisch arbeiten. Und als Geschenk eignet sich das Buch eben besser als der digitale Text. Gerade das Internet hat sich zudem zu einem der wichtigsten Buchmärkte entwickelt. Die interaktiven Seiten mit Schrift, Bild, Audio- und Videoclips haben keineswegs das Interesse am Inhalt zu schmälern vermocht. Die OnlinePiraterie, das Kopieren und Zitieren von Internettexten ohne Angabe der Herkunft und des Autors, die Frage des Autorenrechts im Internet also, ist jedoch nach wie vor ein Problem.

${ }^{13}$ Georgi Čobanov, Literaturata v internet kato institucija, in: Въlgarski ezik i literatura (elektronna versija), 1999, Nr. 5, via LiterNet > E-spisanie BEL (25. 10. 2006).

14 Stanislava Georgieva, Martin Mitov - narodnijat buditel ot Internet. 33-godišnijat poligrafist vkara bъlgarskata literatura v mrežata ot ednata ljubov kъm četeneto, in: Vestnik Sega. Internet Izdanie, 26. 01. 2002; Marija Stankova, Ricarjat na elektronnoto slovo, in: Literaturen forum, br. 14 (498), 9. 04. 2002 - 15. 04. 2002; Čovek ot naroda. Martin, kojto obgrižva bъlgarskata literatura vmesto dъržavata, in: Vestnik Sega. Internet Izdanie, 30. 07. 2004. Alle Artikel sind zugänglich über die website von Martin Mitov: mmm.slovar. org.uk (25. 10. 2006).

15 Der Roman ist zugänglich über den Link, avtori‘ in Slovoto. 
IV

LiterNet, Slovoto, Chulite zeigen in ihrem Design eine durchaus modern-liberale Unübersichtlichkeit und Pluralität. Ruft man etwa Mitovs Link „ljubimi vrъzki“ auf, so gelangt man u. a. zu ,Insajt', einem Journal für populäre Psychologie, zur Bibel, zur Thrakologie, zu mehreren Zeitschriften, zum ,Foto Forum', zur website der bulgarischen Lesben, zur Akademie der Wissenschaften, zum bulgarischen Präsidenten usw. usw. Ein ganz anderes Bild bietet sich jedoch, wenn man in der Liste der Autoren von Slovoto auf den „Bъlgarski inok“ klickt; man wird zu der Gedichtsammlung „Plačevni napevi za rodnija kraj i dušata“ geleitet, die tatsächlich nur das bieten, was der Titel ankündigt, nämlich langweilige patriotische Lyrik, in der ,Rodina', ,Bъlgarija' und ,Bog' meist mit großem Anfangsbuchstaben geschrieben sind. ${ }^{16}$ Anders als sonst bei den Autoren üblich, sind auf diesem Link keine „Biografični beležki““ zu finden, wohl aber eine Werbung für den „Pravoslaven dom“"17, von wo aus man auch die elektronische Bibliothek „Pravoslavna beseda“ erreicht, auf der man eine Reihe orthodoxer Schriften, Biographien von Geistlichen, den Kirchenkalender, Gebete, Heiligenviten, eine orthodoxe Kirchengeschichte, die Zeitschrift der bulgarischen Altgläubigen „Pravoslavno Slovo“ oder auch die Publikation „Mnogostradalna narod", eine Dokumentation über die bulgarischen Christen unter türkischer Herrschaft, aufrufen kann. Wenn man sich von hier zum Buch des Archimandriten Lazar „Grechъt i pokajanieto v poslednite vremena“ weiterleiten lässt, erfährt man, dass Rockmusik, Drogen, Esoterik und östliche Kulte die häufigsten sündigen Zerstreuungen der Gegenwart sind. ${ }^{18}$ Auf den Sites der orthodoxen Kirche geht es nicht um säkulare Bildung, Literatur, Events, Selbstverwirklichung und dergleichen, sondern um den Glauben und die Bewahrung der christlich-orthodoxen Familie, die in der neuheidnischen Gegenwart bedroht sind. Erwartungsgemäß zeigt sich die Kirche nicht liberal, sondern konservativ; das Internet wird hier nicht als Zentrum und Symbol des neuen digitalen Zeitalters, sondern als Medium der Missionierung begriffen.

Durch das Internet ist die bulgarische Literatur Teil eines weltweiten Systems der Information geworden; ob sie jedoch damit auch über den bisherigen Kreis ihrer Rezipienten hinaus wahrgenommen wird, lässt sich nur schwer feststellen. Allerdings dürfte es wenig wahrscheinlich sein, dass der Kreis der Bulgarischlesenden sich spürbar erweitert hätte.

${ }^{16}$ Letzter Zugriff: 19. 10. 2006

$17 \mathrm{http}: / /$ pravoslavie.domainbg.com.

18 Pravoslavna beseda > Pravoslaven duchoven život > Archimandrit Lazar (Abašidze) (25. 10. 2006). Elektronische Version des Buches „Grechъt i pokajanieto v poslednite vremena“, Sofia 1998, aus dem Russ. 
A b s t r a c t: Bulgarian Literature and the Internet. Simultaneously with the fall of the Soviet Empire, politically controlled information came to an end in Bulgaria. A wide variety of literature is now offered by various providers, such as the Academy of Sciences, LiterNet, Slovoto, Chulite, and Pravoslavna Beseda. Thanks to the Internet, the Bulgarian literary scene has turned out to be pluralistic and liberal. If Georgi Chobanov takes the Internet to be the medium which will deliver the user from traditional authorities, Martin Mitov holds the view that the 'old' book and the 'new' Internet are reciprocal.

K e y w ord s : Literature, Internet, Pluralism

Ludger Udolph

Technische Universität Dresden

Institut für Slavistik

Zeunerstr. 1d, 01069 Dresden, Deutschland

Ludger.Udolph@tu-dresden.de 